\title{
Do treatment protocols improve end results? A study of survival of patients with multiple myeloma in Finland
}

\author{
Sakari Karjalainen, Ilmari Palva
}

\begin{abstract}
Objective-To determine whether patients with multiple myeloma treated in three consecutive clinical trials of chemotherapy of the Finnish Leukaemia Group during 1979-85 had a more favourable prognosis than both patients treated in the trial area before the trials and those treated in the rest of Finland.
\end{abstract}

Design-Comparison of the time trend in survival of patients living in the trial area with that of patients living in the rest of Finland.

Setting-Trial area covered 17 of the 21 main hospital districts in Finland (serving more than two thirds of patients with multiple myeloma).

Patients-663 Men and 690 women in the trial area and 318 men and 307 women in the reference area aged under 71 in whom multiple myeloma was diagnosed during 1959-85. In the trial area the disease was diagnosed in 455 men and 493 women in 1959-78 and in 208 men and 197 women in 1979-85; in the reference area it was diagnosed in 234 men and 227 women in 1959-78 and in 84 men and 80 women in 1979-85.

Main outcome measures-Five year cumulative relative survival rates during $1959-85$, annual relative survival rates in the first seven years of follow up during 1979-85, and fitted annual relative survival rates in the first five years of follow up during 1959-85.

Results-During the first two years of follow up the annual relative survival rates did not differ between the two areas, but in the next five years of follow up patients in the trial area did better than those in the reference area. For cases diagnosed in 1979-85 the difference in the five year cumulative relative survival rates between patients in the trial area and those in the reference area was $10 \%$, those in the trial area doing better. Generalised proportional hazards regression analysis of the first five years of follow up showed that the patients in the trial area had a survival advantage over those in the reference area. The model with the best fit included year of follow up, time of diagnosis, the joint effect of year of follow up and time of diagnosis, and the joint effect of area and time of diagnosis.

Conclusion-The patients in the trial area

BrMedf 1989;299:1069-72

TABLE I-Description of clinical trials of treatment of multiple myeloma in Finland in 1979-85

\begin{tabular}{|c|c|c|c|c|c|}
\hline Trial & $\begin{array}{l}\text { Patient } \\
\text { enrolment } \\
\text { (year) }\end{array}$ & $\begin{array}{c}\text { Nature } \\
\text { of } \\
\text { trial }\end{array}$ & $\begin{array}{l}\text { Chemotherapy } \\
\text { regimens }\end{array}$ & $\begin{array}{c}\text { No } \\
\text { of } \\
\text { patients }\end{array}$ & $\begin{array}{c}\text { Median } \\
\text { survival time } \\
(\text { months })\end{array}$ \\
\hline Myeloma $79^{n}$ & Jan 79-Feb 80 & One armed & MOCCA & 50 & 49 \\
\hline Myeloma $80^{7}$ & Mar 80-Jul 82 & Randomised & $\left\{\begin{array}{l}\text { MOCCA } \\
\text { MP }\end{array}\right.$ & $\begin{array}{l}64 \\
66\end{array}$ & $\begin{array}{l}41 \\
45\end{array}$ \\
\hline Myeloma 83 & Jan $83-F e b 86$ & Randomised & $\left\{\begin{array}{l}\text { MOCCA } \\
\text { COLA }\end{array}\right.$ & $\begin{array}{l}76 \\
62\end{array}$ & $\begin{array}{l}\text { Not yet available } \\
\text { Not yet available }\end{array}$ \\
\hline
\end{tabular}

MOCCA $=$ Vincristine, cyclophosphamide, lomustine, melphalan, and meprednisone every fifth week $M P=$ Melphalan and prednisone every fourth week.

COLA $=$ MOCCA but with methylprednisolone omitted after first course of treatment. benefited from the clinical trials, which suggests that the use of a treatment protocol improves the end results of treatment. In other words, the results favour a systematic treatment schedule in preference to a schedule determined by the free choice of a clinician.

\section{Introduction}

One of the principles of clinical practice is that clinicians themselves are free to decide how to treat their patients. There are only a few ethical issues restricting this freedom: clinicians have to take into account the scientific knowledge about the treatment of the condition in question and the wishes and needs of the patient. Health authorities, on the other hand, greatly encourage the development of treatment protocols. The consensus development programme, dating back to 1977 in the United States, ' is the ultimate stage in this process. In the 1980s consensus conferences were also held in some European countries. ${ }^{2-4}$ Treatment protocols make the resource planning and efficiency evaluation easier. The fundamental justification for the use of these protocols is, however, that the effectiveness of the treatment is better with general protocols than without.

Clinical trials include one or more special types of treatment protocols. If successful they may result in more generalised protocols. During 1979-85 the Finnish Leukaemia Group carried out three consecutive clinical trials on the treatment of multiple myeloma. ${ }^{67}$ The study area covered 17 of the 21 hospital districts of the country and more than two thirds of all patients with multiple myeloma in Finland.

With the patient files of the nationwide and population based Finnish Cancer Registry it was possible to study the differences in survival of patients with multiple myeloma in the trial area and the rest of Finland. The purpose of the study was to evaluate the survival differences between these two areas both during and before the trials.

\section{Subjects and methods}

In this study the clinical trials on treatment of multiple myeloma represent an intervention, whose effect was evaluated by comparing the time trend in survival of patients living in the study area with that of patients living in the rest of Finland.

\section{CLINICAL TRIALS ON TREATMENT OF MULTIPLE} MYELOMA IN FINLAND

During 1979-85 three different trials were carried out consecutively in a defined area based on the districts of main hospitals. ${ }^{67}$ The numbers of participating patients, the regimens used, and other details are shown in table I.

\section{FINNISH CANCER REGISTRY}

The Finnish Cancer Registry was founded in 1952. 
Hospitals, medical practitioners, and institutions with hospital beds notify the registry about all cases of cancer that come to their attention. Moreover, pathology laboratories send information on all tissue and cytology specimens with malignant cells. The Central Statistical Office sends information whenever cancer is mentioned on the death certificate. On average five notifications per case are received during the various phases of the disease. Checks have indicated that the registration has been virtually complete. ${ }^{8}$

\section{INCIDENCE OF MULTIPLE MYELOMA IN FINLAND}

During 1970-9 the age standardised ("world standard population") annual incidence of multiple myeloma was $2 \cdot 6$ per 100000 person years in men and $2 \cdot 1$ in women. ${ }^{9}$ The incidence in the 12 provinces of Finland varied from 1.6 to 3.5 in men and from 1.1 to 2.7 in women. There was an increase in the incidence from the 1950 s that slowed down in the 1970s. ${ }^{10}$

\section{PATIENTS AND THEIR FOLLOW UP}

In the clinical trials the disease had to fulfil the diagnostic criteria of multiple myeloma set by the South West Oncology Group. ${ }^{11}$ Emphasis was placed on including only patients with symptoms (painful bone lesions, recurrent infections) or clearly progressive disease. To be included in a trial the patient also had to be previously untreated (for myeloma) and under 71 years of age. In the reference area the diagnostic criteria were the same but probably not followed as systematically as in the trial area.

Our study was of all patients under 71 years of age with multiple myeloma diagnosed in Finland in 195985 and reported to the registry. Of the 2101 patients in the original sample, $123(5 \cdot 9 \%)$ were excluded from the survival analysis as the diagnosis was first known at autopsy or was notified to the registry only from a death certificate. The number of patients included in the survival analysis was 1353 in the study area and 625 in the reference area. The proportions of excluded cases were $5 \cdot 4 \%(n=77)$ and $6.9 \%(n=46)$, respectively.

The patients were followed up for death and emigration through files of the Central Statistical Office of Finland. The follow up came to an end either with the death of the patient or on 31 December 1987, whichever occurred first. Only one patient was lost from follow up because of emigration.

\section{STATISTICAL METHODS}

The proportion of deaths from causes other than multiple myeloma increases with patients' ages. The mortality from other causes is also higher for men than

TABLE II -Numbers and mean ages of patients with multiple myeloma under 71 years of age in Finland in 1959-85 by area, sex, and time of diagnosis

\begin{tabular}{|c|c|c|c|c|c|c|c|c|}
\hline \multirow[b]{3}{*}{ Time of diagnosis } & \multicolumn{4}{|c|}{ Trial area } & \multicolumn{4}{|c|}{ Reference area } \\
\hline & \multicolumn{2}{|c|}{ No of patients } & \multicolumn{2}{|c|}{ Mean age (years) } & \multicolumn{2}{|c|}{ No of patients } & \multicolumn{2}{|c|}{ Mean age (years) } \\
\hline & Men & Women & Men & Women & Men & Women & Men & Women \\
\hline $1959-78$ & 455 & 493 & $58 \cdot 7$ & $59 \cdot 7$ & 234 & 227 & $59 \cdot 5$ & $60 \cdot 0$ \\
\hline $1979-85$ & 208 & 197 & $60 \cdot 9$ & $61 \cdot 4$ & 84 & 80 & $59 \cdot 4$ & $61 \cdot 1$ \\
\hline Total & 663 & 690 & $59 \cdot 4$ & $60 \cdot 2$ & 318 & 307 & $59 \cdot 5$ & $60 \cdot 3$ \\
\hline
\end{tabular}

TABLE III - Numbers and five year (cumulative) relative survival rates in patients with multiple myeloma under 71 years of age in Finland in 1959-85 by time of diagnosis and area

\begin{tabular}{|c|c|c|c|c|c|c|}
\hline \multirow[b]{3}{*}{ Area } & \multicolumn{6}{|c|}{ Time of diagnosis } \\
\hline & \multicolumn{3}{|c|}{$1959-78$} & \multicolumn{3}{|c|}{$1979-85$} \\
\hline & $\begin{array}{c}\text { No } \\
\text { of } \\
\text { patients }\end{array}$ & $\begin{array}{l}\text { Five year relative } \\
\text { survival rate } \\
(\%)\end{array}$ & $\begin{array}{c}95 \% \\
\text { Confidence } \\
\text { interval }\end{array}$ & $\begin{array}{c}\text { No } \\
\text { of } \\
\text { patients }\end{array}$ & $\begin{array}{l}\text { Five year relative } \\
\text { survival rate } \\
(\%)\end{array}$ & $\begin{array}{c}95 \% \\
\text { Confidence } \\
\text { interval }\end{array}$ \\
\hline Trial & 948 & $23 \cdot 9$ & 20.9 to 26.9 & 405 & $37 \cdot 8$ & 32.0 to 43.6 \\
\hline Reference & 461 & $27 \cdot 8$ & $23 \cdot 2$ to $32 \cdot 4$ & 164 & $27 \cdot 5$ & $19 \cdot 2$ to $35 \cdot 8$ \\
\hline
\end{tabular}

women and varies according to five year periods. Because multiple myeloma constitutes a negligible proportion of all deaths in the general population the mortality in the patients from causes other than multiple myeloma can be well estimated as the total mortality in comparable persons in the general population. From death rates in the general population an expected survival rate can be calculated for the patients for each follow up year separately and cumulatively, for example, over five years. For a given patient group an expected survival rate ${ }^{1213}$ is derived as an average of the patient specific survival probabilities adopted from the general population life tables defined according to the time of the year, sex, and age. ${ }^{14-19}$ Annual probabilities of the patients alive at the beginning of the annual interval are used to calculate annual expected rates. For cumulative five year expected rates patient specific five year probabilities are averaged over all patients alive at the beginning of the follow up - that is, at diagnosis.

The relative survival rate ${ }^{20}$ is the ratio between the observed and expected survival rate. Both annual and five year cumulative relative survival rates are calculated. When the relative survival rate is equal to 1.00 -that is, $100 \%$-patients do not have excess mortality attributable to their disease. Thus when the annual relative survival rates reach $100 \%$ the patients may be considered to be cured and the follow up year in question may be called the point (year) of cure. The annual relative survival rates in the first seven years of follow up during 1979-85 were plotted to show the excess risk of dying in relation to time from diagnosis. The relative survival rates have been widely used in cancer statistics and research..$^{21-23}$

The effect of sex, time of diagnosis, and area of residence as prognostic factors upon the relative survival was evaluated with an extension of the Cox proportional hazards model..$^{24}$ This model is exactly the same as the traditional proportional hazards model, but instead of observed survival rates it is applied to the relative survival rates. The model was fitted to the data with the method described by Hakulinen and Tenkanen, ${ }^{24}$ based on annual follow up intervals and making use of the statistical package generalised linear interactive modelling (GLIM). ${ }^{25}$ This method of analysis also facilitates the study of the basic proportionality assumption as interaction terms involving the follow up year. The differences in deviance between hierarchical models were compared with corresponding differences in the degrees of freedom with the $\chi^{2}$ distribution.

All variables were categorised. There were two classes of area of residence: the trial area and reference area (the rest of Finland). The time of diagnosis was also divided into two classes: 1959-78 and 1979-85. The effect of the variables is shown as fitted annual relative survival rates based on the model with the best fit.

\section{Results}

There were almost equal numbers of male and female patients (table II). The women were slightly older than the men. The small difference in the sex distributions and mean ages between the areas is most probably due to random variation. The information on the verification of the diagnosis was missing in $4 \cdot 8 \%$ (65) of the cases in the trial area and in $5 \cdot 7 \%$ (36) of the cases in the reference area.

For cases diagnosed in 1979-85 the five year cumulative relative survival rate in patients living in the trial area was $10 \%$ higher than that in patients living in the reference area, being $37 \cdot 8 \%$ and $27 \cdot 5 \%$ respectively (table III). There was a trend of improving survival rates in the trial area but not in the reference 


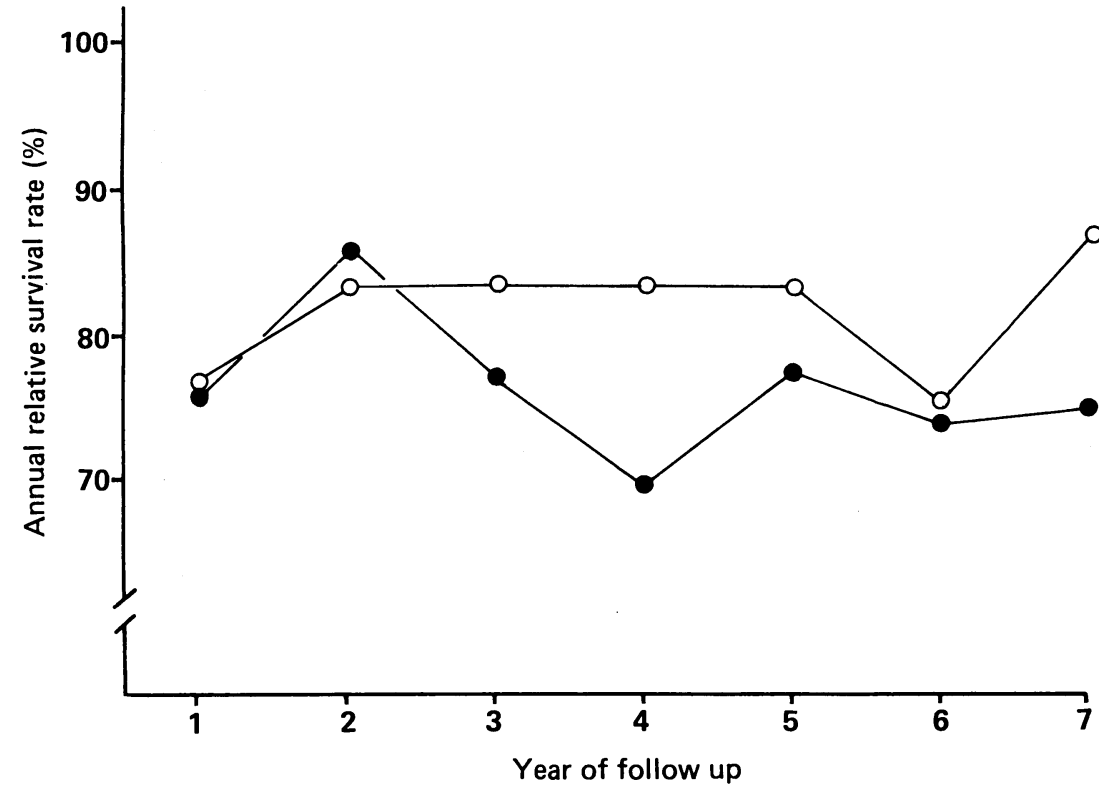

FIG 1-Annual relative survival rates in trial area $(\mathrm{O}-\mathrm{O})$ and first seven years of follow up of patients under 71 with multiple myeloma diagnosed in Finland in 1979-85

area. The difference between the five year cumulative relative survival rate of patients in the trial area and that of patients in the reference area in 1959-78 was $-3.9 \%$ ( $95 \%$ confidence interval -9.4 to 1.6$)$, respectively, and in $1979-85$ it was $10.3 \%(0.4$ to $20 \cdot 2)$. reference area $(\bullet \bullet)$ during

For cases diagnosed in $1979-85$ the annual relative survival rate did not reach $100 \%$, or even $90 \%$, during the first seven years of follow up (fig 1). There was no difference in the annual relative survival rate between the two areas during the first two years of follow up. During the next five years of follow up patients living in the trial area did better, which also explains the difference in the five year relative survival rates (table III) for the same period.

Table IV describes the steps in fitting the generalised proportional hazards models for the first five years of follow up. The model with the best fit included the year of follow up and time of diagnosis, the joint effect of these two variables, and the joint effect of area of residence and time of diagnosis (model 8). The area as such did not affect the relative survival rate (models 5 and 7).

The fitted annual relative survival rates (based on model 8 , table IV) show the change by time of

TABLE IV-Results of fitting generalised linear models to annual relative survival rates during first five years of follow up in patients under 71 with multiple myeloma diagnosed in Finland in 1959-85

\begin{tabular}{lcccc}
\hline & & \multirow{2}{*}{$\begin{array}{c}\text { Degrees } \\
\text { Model }\end{array}$} & \multicolumn{2}{c}{ Difference in: } \\
\cline { 5 - 5 } & Deviance & freedom & Deviancet & Degrees of freedom \\
\hline 1: Constant & $223 \cdot 0$ & 39 & & \\
2: Model 1 + follow up year & $89 \cdot 2$ & 35 & $133 \cdot 8^{\star \star \star}$ & 4 \\
3: Model 2+ sex & $86 \cdot 8$ & 34 & $2 \cdot 4$ & 1 \\
4: Model 2+ period & $50 \cdot 7$ & 34 & $38 \cdot 5 \star{ }^{\star \star \star}$ & 1 \\
5: Model 4+ area & $50 \cdot 3$ & 33 & $0 \cdot 4$ & 1 \\
6: Model 4+ period.(follow up year) & $29 \cdot 7$ & 30 & $21 \cdot 0^{\star \star \star}$ & 4 \\
7: Model 6+area & $29 \cdot 5$ & 29 & $0 \cdot 2$ & 1 \\
8: Model 7+area.period & $25 \cdot 6$ & 28 & $3 \cdot 9^{\star}$ & 1 \\
& & & & \\
\hline
\end{tabular}

†Compared with the model to which the last term has been added; significance of term inclusion: ${ }^{\star} 0 \cdot 01<\mathrm{p}<0 \cdot 05$ $\star \star \star p<0 \cdot 001$

TABLE $\mathrm{V}-$ Trends in age adjusted incidence of multiple myeloma (per 1000000 person years) in Finland in 1959-85 by area, sex, and time of diagnosis

\begin{tabular}{lccc}
\hline & \multicolumn{3}{c}{ Time of diagnosis } \\
\cline { 2 - 4 } & \multicolumn{4}{c}{$1959-68$} & $1969-78$ & $1979-85$ \\
\hline \multicolumn{4}{c}{ Trial area } \\
Men & 17 & 29 & 28 \\
Women & 13 & 25 & 24 \\
& \multicolumn{2}{c}{ Reference area } & \\
Men & 23 & 29 & 27 \\
Women & 17 & 20 & 20 \\
\hline
\end{tabular}

diagnosis in the excess risk of dying of multiple myeloma between patients living in the two areas, showing the survival advantage gained by the patients of the trial area (fig 2). The patients in whom the disease was diagnosed in 1979-85 had a higher relative survival rate during the first three years of follow up but not in the subsequent two years than patients in whom it was diagnosed earlier.

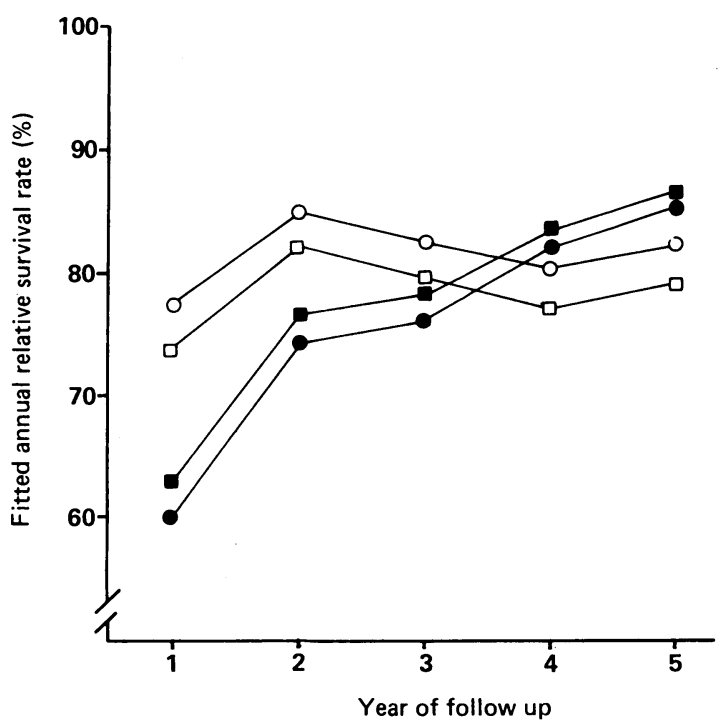

FIG 2-Fitted annual relative survival rates during five years of follow up of patients under 71 with multiple myeloma diagnosed in Finland in 1979-85 in trial area $(\bullet-\bullet)$ and reference area (- ) and in 1979-85 in trial area $\left(\mathrm{O}_{-} \mathrm{O}\right)$ and reference area ( $\left.\square-\square\right)$. Data based on model 8 in table IV

\section{Discussion}

In principle, the choice between various methods of treatment should be based on randomised clinical trials. $^{5}$ The treatment procedure in a trial has two elements that may affect the result: the regimens used and the protocol itself. The protocol tells not only which regimens should be used but also, for example, when the various treatment phases will take place, how the follow up of patients should be organised, and how to deal with possible side effects and relapses. The same scientific principles should be applied to the decision whether to use protocols as are applied to the choice of regimen. To our knowledge no randomised clinical trials have been performed in which only the effect of protocol has been measured. Our aim was to assess whether the use of protocols makes the treatment more effective than that based on the individual choices of clinicians.

In Finland there were three consecutive clinical trials $^{67}$ in $1979-85$ on treatment of patients with multiple myeloma in an area that included two thirds of all patients in Finland. The studies were population based - that is, all new patients with myeloma in the area were included if eligible according to the study protocol. By using the patient files of the Finnish Cancer Registry it was possible to study differences in survival of patients by area (in Finland the place of residence decides where the patient will be examined and treated). Of the 405 registered patients in the trial area, $318(76 \%)$ participated in the trials (tables I and II). One or two strict treatment protocols were used continuously. In other parts of Finland the treatment was based on the free choice of the clinicians. Generally, treatment in the reference area followed these guidelines: the primary choice was to use intermittent courses of melphalan and prednisone and the secondary choice was aggressive combination chemotherapy. The trial protocols were sent also to the hospitals of the non-participating districts. We presumed that the 
main difference between the two areas was not in the regimens themselves but in the level of uniformity of the treatment (including follow up).

During the time of the trials, 1979-85, the patients living in the trial area had a higher five year relative survival rate than the patients living in other parts of Finland $(37 \cdot 8 \% v 27 \cdot 5 \%)$. Before the trial the results were the opposite: patients in the reference area had a higher survival rate. This could imply that the patients treated by trial protocols gained a real advantage, which is further supported by the results of regression analysis (table IV and fig 2).

The significant difference in survival between the patients living in the two regions has two explanations: confounding or a real effect. Firstly, the list of possible confounders includes sex, age, and diagnostic procedures. The sex variable did not improve the fit in regression analysis, and there seemed to be no large differences in the mean ages of patients. If there had been a systematic variation in diagnostic procedures a more active search for new cases and more sensitive diagnostic methods would have resulted in earlier diagnosis and, thus, in improved survival rates in the trial area. This would have happened with or without real improvement in the effectiveness of treatment. We evaluated this possibility by comparing the incidences of multiple myeloma by time of diagnosis and area (table V). No important changes were seen in the diagnosis of multiple myeloma when the trials were introduced. It is quite improbable that any of these confounders could explain the significant difference in survival.

Secondly, the patients in the trial area had a survival advantage either because of the use of protocols or because the more effective regimens were used in the trial area. During the second trial the experimental treatment was less effective than the reference treatment,' which was also the main regimen in the reference area. This implies that the use of protocols is the more reasonable explanation for the survival advantage. Furthermore, the less effective trial regimen may have diminished the difference between the areas. The physicians committed to the trials may have become more experienced. They were supervised by experienced haematologists in the area. Also the mutual exchange of experience was probably more active between clinicians in the trial area than between those in the reference area. The training effect of trials may explain part of the difference in the end results of treatment.

The survival advantage gained by the patients in the trial area was not immediate. There was no difference in survival between the two patient groups during the first two years of follow up (fig 1), but during the next five years the patients in the trial area had higher annual relative survival rates. There are at least two explanations for the delayed effect. Firstly, the survival is calculated from the diagnosis but in myeloma the treatment is often started much later. Secondly, even if patients are treated equally at the beginning there is more variation in treatment in the later phases of the disease. The (trial) protocol gives rules for how to deal with relapses and certain types of treatment side effects. Both of these explanations seem to be reasonable and would favour the use of protocols.

The fitted annual relative survival rates based on the model with the best fit in the regression analysis (model 8 , table IV) show not only the survival advantage gained by the patients in the trial area but also that patients in whom the disease was diagnosed in 1979-85 had a lower risk of dying of multiple myeloma during the first and third years of follow up than patients in whom the disease was diagnosed earlier (fig 2). This means that, although the difference between the two areas appeared during the later years of follow up, the improvement in survival according to time of diagnosis was mainly due to more effective treatment during the first three years after diagnosis. During the fourth and fifth years of follow up the results were the opposite (fig 2). In this case the deaths due to the malignant disease could be postponed but not totally avoided, which may well be also because of earlier diagnosis. This difference is, however, based on only a few deaths.

In conclusion, the difference in five year relative survival rate in patients with myeloma between the trial and reference area was more than $10 \%$. The difference was significant, which implies that the results support a systematic treatment schedule in preference to the free choice of a clinician. It is, however, not definitely clear that the effects of trial protocols can be regarded as a good representation of treatment protocols in general. Participation in clinical trials is voluntary, and practitioners may regard other kinds of protocols as unnecessary and restrictive, leading to a much lower compliance in the use of protocols. The correct way to draw any further conclusions about the effectiveness of the use of protocols is to run a clinical trial in which the only difference is in the use of a protocol.

1 Office for Medical Applications of Research. NIH consensus developmen conference summaries. Vol 2. Bethesda, Maryland: Office for Medical Applications of Research, 1979.

2 Agersnap T, Jakobsen G, Kempinski J. Konsensuskonferencer i Danmark [in Danish]. Copenhagen: Danish Hospital Institute, 1984.

3 Consensus development conference: coronary artery bypass grafting. $\mathrm{BrMed} \mathcal{f}$ 1984;289:1690-1.

4 Jacoby I, Rose M. Consensus conference on cholesterol and heart disease. Lancet 1985;ii:205.

5 Cochrane AL. Effectiveness and efficiency. Random reflections on health services. Abingdon, Berkshire: Nuffield Provincial Hospitals Trust, 1972.

6 Finnish Leukaemia Group. Aggressive combination chemotherapy in multiple myeloma. A multicentre trial. Scandinavian foumal of Haematolog 1985;35:205-9.

7 Finnish Leukaemia Group. Treatment of multiple myeloma with an intensive 5-drug combination or intermittent melphalan and prednisone; a randomised multicentre trial. Eur f Haematol 1987;38:50-4.

8 Saxén E, Teppo L. Finnish Cancer Registry. Twenty-five years of a nationwide cancer registry. Helsinki: Finnish Cancer Registry, 1978:1-47.

9 Jensen OM, Carstensen B, Glattre E, Malker B, Pukkala E, Tulinius H. Atlas of cancer incidence in the Nordic countries. Helsinki: Nordic Cancer Union, 1988:177-81.

10 Hakulinen T, Andersen A, Malker B, Pukkala E, Schou G, Tulinius H. Trends in cancer incidence in the Nordic countries: a collaborative study of the five Nordic cancer registries. Acta Pathol Microbiol Immunol Scand [A] 1986;94(suppl 288):90-1.

11 Durie BMG, Salmon SE. Multiple myeloma, macroglobulinaemia and monoclonal gammopathies. In: Hoffbrand AV, Brain HC, Hirsh J, eds. Recent advances in haematology 2. Edinburgh: Churchill Livingstone, Recent advances

12 Ederer F, Axtell LM, Cutler SJ. The relative survival rate: a statistical methodology. National Cancer Institue Monographs 1961;6:101-21.

13 Hakulinen T. Cancer survival corrected for heterogeneity in patient with drawal. Biometrics 1982;38:933-42.

14 Central Statistical Office of Finland. Mortality: life tables 1956-60. Helsinki Central Statistical Office of Finland, 1963. (Official Statistics of Finland VI A:121.)

15 Central Statistical Office of Finland. Mortality: life tables 1961-5. Helsinki Central Statistical Office of Finland, 1968. (Official Statistics of Finland VI A:126.)

16 Central Statistical Office of Finland. Mortality: life tables 1966-70. Helsinki: Central Statistical Office of Finland, 1974. (Official Statistics of Finland VI A:134.)

17 Central Statistical Office of Finland. Mortality: life tables 1971-5. Helsinki: Central Statistical Office of Finland, 1980. (Official Statistics of Finland VI A:142.

18 Central Statistical Office of Finland. Mortality: life tables 1976-80. Helsinki Central Statistical Office of Finland, 1984. (Official Statistics of Finland VI A:147.)

19 Central Statistical Office of Finland. Mortality: life tables 1981-5. Helsinki: Central Statistical Office of Finland, 1987. (Official Statistics of Finland VI A:152.)

20 Hakulinen T. On long-term relative survival rates. 7 Chronic Dis 1977;30 431-43.

21 Office of Population Censuses and Surveys. Cancer statistics-survival. Survival up to 1980 of cases diagnosed cancer registered in England and Wales during 1971-5. London: HMSO, 1982. (Series MB1 No 9.)

22 Cancer Research Campaign. Trends in cancer survival in Great Britain. Cases registered between 1960 and 1974. London: Cancer Research Campaign, 1982.

23 Adami H-O, Malker B, Holmberg L, Persson I, Stone B. The relation between survival and age at diagnosis in breast cancer. $N$ Engl $f$ Med 1986;315: $559-63$.

24 Hakulinen T, Tenkanen L. Regression analysis of the relative survival rates. Applied Statistics 1987;36:307-17.

25 Payne $\mathrm{CD}$, ed. The GLIM system release 3.77. Generalized linear interactive modelling manual. Oxford: Numerical Algorithms Group, 1986:1-183.

(Accepted 4 September 1989) 